Pure Mathematical Sciences, Vol. 2, 2013, no. 2, 83 - 88

HIKARI Ltd, www.m-hikari.com

\title{
Improvements of Some Principal Results in Abstract Duality
}

\author{
WonSok Yoo \\ Department of Applied Mathematics \\ Kumoh National Institute of Technology \\ Kumi 730-701, Korea \\ wsyoo@kumoh.ac.kr
}

Copyright (c) 2013 WonSok Yoo. This is an open access article distributed under the Creative Commons Attribution License, which permits unrestricted use, distribution, and reproduction in any medium, provided the original work is properly cited.

\begin{abstract}
In this note, we would like to establish some improved results, which are basic principle in analysis, of the classical Ascoil theorems and give extremely important results of convenient consequences of these results.
\end{abstract}

\section{Mathematics Subject Classification: 54b10}

Keywords: Classical Ascoil theorems, Alaoglu-Bourbaki Theorem, Improved Vitali-Hahn-Saks-Graves-Ruess Theorem, Improved Bartle-DunfordSchwartz-Nikodym Theorem

\section{Introduction}

Let $\Omega$ be a topological space and $X=(X, \mathcal{U})$ a uniform space with the uniformity $\mathcal{U} \subset 2^{\left(X^{2}\right)}$. For $S \subset C(\Omega, X)$, the topology $\tau_{u}$ of uniform convergence on compact sets is equal to the compact open topology $\tau_{k}([2, \mathrm{p} .284])$. Then we have three classical Ascoil theorems as follows.

Theorem A. Let $\Omega$ be locally compact, $X$ a uniform space and $S$ a closed set in $\left(C(\Omega, X), \tau_{k}\right)$. Then $S$ is compact in $\left(C(\Omega, X), \tau_{k}\right)$ if and only if $S$ is equicontinuous and $\{g(\omega): g \in S\}$ is compact in $X$ for each $\omega \in \Omega$ ([2, p.290],[3, Ch. 7]). 
Theorem B. Let $\Omega$ be a Hausdorff $k$ space or regular $k$ space, $X$ a Hausdorff uniform space and $S \subset C(\Omega, X)$. Then $S$ is compact in $\left(C(\Omega, X), \tau_{k}\right)$ if and only if

( $\alpha) S$ is closed in $\left(C(\Omega, X), \tau_{k}\right)$,

( $\beta) S$ is equicontinuous on each compact set in $\Omega$,

$(\gamma) \overline{\{g(\omega): g \in S\}}$ is compact for each $\omega \in \Omega([3$, Ch.7 $])$.

Theorem C. Let $\Omega$ be a compact space, $X$ a pseudometric space and $S \subset$ $C(\Omega, X)$. Let $u \Omega$ be the topology on $C(\Omega, X)$ for which $f_{\alpha} \stackrel{u \Omega}{\longrightarrow} f$ if and only if $\lim _{\alpha} f_{\alpha}(\omega)=f(\omega)$ uniformly for $\omega \in \Omega$. Then $S$ is compact in $(C(\Omega, X), u \Omega)$ if and only if

$\left(\alpha^{\prime}\right) S$ is closed in $(C(\Omega, X), u \Omega)$,

$\left(\beta^{\prime}\right) S$ is equicontinuous on $\Omega$,

$(\gamma) \overline{\{g(\omega): g \in S\}}$ is compact for each $\omega \in \Omega([6$, Ch.6 $])$.

Here comes two problems.

Firstly, a compact space might not be locally compact, and there exist many non-regular compact spaces and non-Hausdorff compact spaces. In fact, if a compact space is not locally compact, then it is neither regular nor Hausdorff. Besides, many uniform spaces are not metrizable while Theorem $\mathrm{C}$ is dealing with the pair $(\Omega, X)$ of compact $\Omega$ and metrizable $X$. Hence we would like to establish a result on a level with Theorem A and B for the pair $(\Omega, X)$ of compact space $\Omega$ and uniform space $X$.

Secondly, for the simplest case of $X=\mathbb{C}$, Theorem $\mathrm{C}$ has an improved version named Arzela-Ascoli theorem: for compact space $\Omega$ and $S \subset C(\Omega, \mathbb{C})$, $S$ is relatively compact in $(C(\Omega, \mathbb{C}), u \Omega)$ if and only if $S$ is equicontinuous and $\{g(\omega): g \in S, \omega \in \Omega\}$ is relatively compact in $\mathbb{C}$, i.e., $\sup _{g \in S, \omega \in \Omega}|g(\omega)|<+\infty$ $([4$, p.266]). Hence we would like to establish a result on a level with the ArzelaAscoli theorem for the pair $(\Omega, X)$ of compact space $\Omega$ and pseudometric space $X$.

\section{Main Results}

Let $X$ be a topological space, $\Omega \neq \varnothing$ and $X^{\Omega}$ the family of $X$-valued mappings defined on $\Omega$. Let $\sigma \Omega$ be the topology on $X^{\Omega}$ such that $f_{\alpha} \stackrel{\sigma \Omega}{\longrightarrow} f$ if and only if $\lim _{\alpha} f_{\alpha}(\omega)=f(\omega)$ at each $\omega \in \Omega$.

Taking $X_{\omega}=X$ for each $\omega \in \Omega$ and $h(f)=(f(\omega))_{\omega \in \Omega}$ for $f \in X^{\Omega}$, one can see that $h: X^{\Omega} \rightarrow \Pi_{\omega \in \Omega} X_{\omega}$ is a homeomorphism between $\left(X^{\Omega}, \sigma \Omega\right)$ and the product space $\Pi_{\omega \in \Omega} X_{\omega}$. So we can say that $\left(X^{\Omega}, \sigma \Omega\right)=\Pi_{\omega \in \Omega} X_{\omega}$ and $f=(f(\omega))_{\omega \in \Omega}$ for all $f \in X^{\Omega}$.

Observe that if $S \subseteq F \subseteq X^{\Omega}$, then

$\bar{S}^{(F, \sigma \Omega)}=\left\{f \in F: \exists \operatorname{net}\left(f_{\alpha}\right)_{\alpha \in(I, \leq)} \subset S\right.$ such that $\left.\lim _{\alpha} f_{\alpha}(\omega)=f(\omega), \forall \omega \in \Omega\right\}$ 
is just the closure of $S$ in $(F, \sigma \Omega)$.

We are now in a position to state and prove the main theorem.

Theorem 2.1. Let $X$ be a topological space, $\Omega \neq \varnothing$ and $S \subseteq F \subseteq X^{\Omega}$. If

(a) $\bar{S}^{\left(X^{\Omega}, \sigma \Omega\right)} \subseteq$ F, i.e., $\bar{S}^{(F, \sigma \Omega)}=\bar{S}^{\left(X^{\Omega}, \sigma \Omega\right)}$ and

(b) $\overline{\{g(\omega): g \in S\}}$ is compact for each $\omega \in \Omega$,

then $\bar{S}^{(F, \sigma \Omega)}$ is compact in $(F, \sigma \Omega)$.

If, in addition, $X$ is Hausdorff, then the converse holds, i.e., $\bar{S}^{(F, \sigma \Omega)}$ is compact in $(F, \sigma \Omega)$ then (a) and (b) hold for $S$.

Proof. Suppose that (a) and (b) hold for $S \subseteq F \subseteq X^{\Omega}$. If $f \in \bar{S}^{(F, \sigma \Omega)}$, then

$$
f(\omega) \in \overline{\{g(\omega): g \in S\}} \text { for each } \omega \in \Omega
$$

and hence

$$
(f(\omega))_{\omega \in \Omega} \in \prod_{\omega \in \Omega} \overline{\{g(\omega): g \in S\}} .
$$

By (a), $\bar{S}^{(F, \sigma \Omega)}=\bar{S}^{\left(X^{\Omega}, \sigma \Omega\right)}$ and so $\bar{S}^{(F, \sigma \Omega)}$ is closed in $\left(X^{\Omega}, \sigma \Omega\right)=\Pi_{\omega \in \Omega} X_{\omega}$, i.e.,

$$
\bar{S}^{(F, \sigma \Omega)}=\left\{(f(\omega))_{\omega \in \Omega}: f \in \bar{S}^{(F, \sigma \Omega)}\right\}
$$

is closed in $\Pi_{\omega \in \Omega} \overline{\{g(\omega): g \in S\}}$. By (b) and the Tychonoff's theorem, $\Pi_{\omega \in \Omega} \overline{\{g(\omega): g \in S\}}$ is compact and so $\bar{S}^{(F, \sigma \Omega)}$ is compact in $\Pi_{\omega \in \Omega} \overline{\{g(\omega): g \in S\}}$. Since

$$
\bar{S}^{(F, \sigma \Omega)} \subset(F, \sigma \Omega) \cap\left(\Pi_{\omega \in \Omega} \overline{\{g(\omega): g \in S\}}\right),
$$

it follows that $\bar{S}^{(F, \sigma \Omega)}$ is compact in $(F, \sigma \Omega)$.

Conversely, suppose that $X$ is Hausdorff and $\bar{S}^{(F, \sigma \Omega)}$ is compact in $(F, \sigma \Omega)$. If $\left(f_{\alpha}\right)_{\alpha \in I}$ is a net in $S$ such that $f_{\alpha} \stackrel{\sigma \Omega}{\longrightarrow} f \in X^{\Omega}$, then there is a subnet $\left(f_{\alpha_{\gamma}}\right)_{\gamma \in J}$ of $\left(f_{\alpha}\right)_{\alpha \in I}$ such that $f_{\alpha_{\gamma}} \stackrel{\sigma \Omega}{\longrightarrow} g \in \bar{S}^{(F, \sigma \Omega)}$ and so $f(\omega)=\lim _{\gamma} f_{\alpha_{\gamma}}(\omega)=$ $g(\omega)$ for all $\omega \in \Omega$ in the Hausdorff space $X$, i.e., $f=g \in \bar{S}^{(F, \sigma \Omega)}$. Thus, $\bar{S}^{\left(X^{\Omega}, \sigma \Omega\right)} \subseteq \bar{S}^{(F, \sigma \Omega)} \subseteq F$, i.e., (a) holds for $S$.

For each $\omega \in \Omega$, let $P_{\omega}:\left(X^{\Omega}, \sigma \Omega\right) \rightarrow X$ with $P_{\omega}(f)=f(\omega)$. Since $P_{\omega}$ is continuous,

$$
\left\{f\left(\omega: f \in \bar{S}^{(F, \sigma \Omega)}\right\}=P_{\omega}\left(\bar{S}^{(F, \sigma \Omega)}\right)\right.
$$

is compact and so it is closed in the Hausdorff space $X$ for each $\omega \in \Omega$. Then $\overline{\{g(\omega): g \in S\}} \subseteq \overline{\left\{f(\omega): f \in \bar{S}^{(F, \sigma \Omega)}\right\}}=\left\{f(\omega): f \in \bar{S}^{(F, \sigma \Omega)}\right\} \subseteq \overline{\{g(\omega): g \in S\}}$ for all $\omega \in \Omega$. Thus,

$$
\overline{\{g(\omega): g \in S\}}=\left\{f(\omega): f \in \bar{S}^{(F, \sigma \Omega)}\right\}
$$

is compact for each $\omega \in \Omega$, i.e., (b) holds for $S$. 
It should be noted that Theorem 2.1 is a basic principle in analysis because many extremely important results are convenient consequences of Theorem 2.1.

Corollary 2.2. (Alaoglu-Bourbaki Theorem). Let $X$ be a locally convex space with the dual $X^{\prime}, S \subset X^{\prime}$. If $S$ is equicontinuous, then $S$ is relatively compact in $\left(X^{\prime}, \sigma X\right)=\left(X^{\prime}\right.$, weak*). If $U$ is a neighborhood of $0 \in X$, then the polar $U^{\circ}=\left\{x^{\prime} \in X^{\prime}:\left|x^{\prime}(u)\right| \leq 1\right.$ for all $\left.u \in U\right\}$ is compact in $\left(X^{\prime}, \sigma X\right)=$ $\left(X^{\prime}\right.$, weak*).

Proof. It is obvious that if $S$ is equicontinuous, then $\sup _{f \in S}|f(x)|<+\infty$ for each $x \in X$ and $\bar{S}^{\left(\mathbb{C}^{X}, \sigma \Omega\right)} \subset X^{\prime}$. If $U$ is a neighborhood of $0 \in X$, then ${\overline{U^{\circ}}}^{\left(\mathbb{C}^{X}, \sigma \Omega\right)}=U^{\circ} \subset X^{\prime}$ and $U^{\circ}$ is equicontinuous.

Corollary 2.3. (Improved Vitali-Hahn-Saks-Graves-Ruess Theorem). Let $X$ be a complete locally convex space and $\Sigma$ a $\sigma$-algebra of subsets of a set. Then for a family of countably additive measures $S \subset c a(\Sigma, X)$, the following (I), (II) and (III) are equivalent.

(I) $S$ is relatively compact in $(c a(\Sigma, X), \sigma \Sigma)$.

(II) $\{\overline{\mu(A): \mu \in S}\}$ is compact for each $A \in \Sigma$ and $\bar{S}^{(c a(\Sigma, X), \sigma \Sigma)}$ is uniformly countably additive, i.e., if $\left\{A_{j}\right\}$ is pairwise disjoint in $\Sigma$, then $\lim _{n} \sum_{j=1}^{n} \mu\left(A_{j}\right)=$ $\mu\left(\cup_{j=1}^{\infty} A_{j}\right)$ uniformly for $\mu \in \bar{S}^{(c a(\Sigma, X), \sigma \Sigma)}$.

(III) $\{\overline{\mu(A): \mu \in S}\}$ is compact for each $A \in \Sigma$ and $\bar{S}^{\left(X^{\Sigma}, \sigma \Sigma\right)} \subset c a(\Sigma, X)$.

Proof. $\bar{S}^{(c a(\Sigma, X), \sigma \Sigma)}$ is abbreviated to $\bar{S}^{\sigma \Sigma}$ in this proof.

$(\mathrm{I}) \Rightarrow(\mathrm{II})$. For $A \in \Sigma$, define $P_{A}:(c a(\Sigma, X), \sigma \Sigma) \rightarrow X$ by $P_{A}(\mu)=\mu(A)$. Then $P_{A}$ is continuous and so $\left\{\mu(A): \mu \in \bar{S}^{\sigma \Sigma}\right\}=P_{A}\left(\bar{S}^{\sigma \Sigma}\right)$ is compact. Since $X$ is completely regular, $\overline{\left\{\mu(A): \mu \in \bar{S}^{\sigma \Sigma}\right\}}$ is compact and $\overline{\{\mu(A): \mu \in S\}} \subset$ $\overline{\left\{\mu(A): \mu \in \bar{S}^{\sigma \Sigma}\right\}}$, and thus $\overline{\{\mu(A): \mu \in S\}}$ is compact.

Since $\bar{S}^{\sigma \Sigma}$ is conpact in $(c a(\Sigma, X), \sigma \Sigma)$, it follows from COR 4.3 of [7] or Th. 7 of [5] that $\bar{S}^{\sigma \Sigma}$ is uniformly countably additive.

$(\mathrm{II}) \Rightarrow(\mathrm{III})$ Suppose $\left\{A_{j}\right\} \subset \Sigma, A_{i} \cap A_{j}=\varnothing(i \neq j)$ and $\left(\mu_{\alpha}\right)_{\alpha \in I}$ is a net in $S$ such that $\mu_{\alpha} \stackrel{\sigma \Sigma}{\longrightarrow} \mu \in X^{\Sigma}$. Since $X$ is complete, $\mu\left(\cup_{j=1}^{\infty} A_{j}\right)=$ $\lim _{\alpha} \mu_{\alpha}\left(\cup_{j=1}^{\infty} A_{j}\right)=\lim _{\alpha} \lim _{n} \sum_{j=1}^{n} \mu_{\alpha}\left(A_{j}\right)=\lim _{n} \lim _{\alpha} \sum_{j=1}^{n} \mu_{\alpha}\left(A_{j}\right)=\lim _{n} \sum_{j=1}^{n} \mu\left(A_{j}\right)=$ $\sum_{j=1}^{\infty} \mu\left(A_{j}\right)$.

$(\mathrm{III}) \Rightarrow(\mathrm{I})$. Theorem 2.1

According to [4, pp.67-68], a set $A$ in a locally convex space $X$ is said to be weakly sequentially compact if every sequence $\left\{x_{n}\right\} \subset A$ contains a subsequence which converges weakly to a point in $X$. 
Corollary 2.4. (Improved Bartle-Dunford-Schwartz-Nikodym Theorem). Let $\Sigma$ be a $\sigma$-algebra of subsets of a set. For $S \subset c a(\Sigma, \mathbb{C})$, the following (1)-(5) are equivalent.

(1) $S$ is weakly sequentially compact in the Banach space $c a(\Sigma, \mathbb{C})$ with the norm of total variation ([4, p.240]).

(2) $S$ is relatively compact in $c a(\Sigma, \mathbb{C}), \sigma \Sigma)$.

(3) $\sup _{\mu \in S}|\mu(A)|<+\infty, \forall A \in \Sigma$; if $\left(\mu_{\alpha}\right)_{\alpha \in I}$ is a net in $S$ such that $\lim _{\alpha} \mu_{\alpha}(A)=\mu(A)$ exists at each $A \in \Sigma$, then $\mu \in c a(\Sigma, \mathbb{C})$.

(4) $\sup _{\mu \in S}|\mu(A)|<+\infty, \forall A \in \Sigma ; S$ is uniformly countably additive.

(5) $\sup _{\mu \in S, A \in \Sigma}|\mu(A)|<+\infty$ and $S$ is uniformly countably additive.

Proof. (1) $\Leftrightarrow$ (5) by Bartle-Dunford-Schwartz-Nikodym theorem ([4, p.305]), $(2) \Leftrightarrow(3)$ by Theorem $2.1,(2) \Rightarrow(5)$ by Corollary 2.3 and the Nikodym theorem ([4, pp.309-310]). As in the proof of (II) $\Rightarrow$ (III) in Corollary 2.3, it is easy to see that $(5) \Rightarrow(4) \Rightarrow(3)$. Thus, $(1) \Rightarrow(5) \Rightarrow(4) \Rightarrow(3) \Rightarrow(2) \Rightarrow(5) \Rightarrow(1)$.

A Banach space is reflexive if $X=X^{\prime \prime}$, the dual of the dual $X^{\prime}$.

Corollary 2.5. A Banach space $X$ is reflexive if and only if $B=\{x \in X$ : $\|x\| \leq 1\}$ is compact in $(X$, weak $)=\left(X, \sigma X^{\prime}\right)$.

Proof. Observe that $B \subset X \subset \mathbb{C}^{X^{\prime}}$. If $f \in \bar{B}^{\left(\mathbb{C}^{X^{\prime}}, \sigma X^{\prime}\right)}$, then there is a net $\left(x_{\alpha}\right)_{\alpha \in I}$ in $B$ such that $x_{\alpha} \stackrel{\sigma X^{\prime}}{\longrightarrow} f$ and hence $\left|f\left(x^{\prime}\right)\right|=\lim _{\alpha}\left|x_{\alpha}\left(x^{\prime}\right)\right| \leq$ $\sup _{\alpha \in I}|| x_{\alpha}\|\| x^{\prime}\|\leq\| x^{\prime} \|, \forall x^{\prime} \in X^{\prime}$, i.e., $f \in X^{\prime \prime}$.

If $X$ is reflexive, then $\bar{B}^{\left(\mathbb{C}^{X^{\prime}}, \sigma X^{\prime}\right)} \subset X^{\prime \prime}=X$. Since $\sup _{x \in B}\left|x\left(x^{\prime}\right)\right|=\left\|x^{\prime}\right\|<$ $+\infty$ for each $x^{\prime} \in X^{\prime}$, it follows from Theorem 2.1 that $B$ is compact in $\left(X, \sigma X^{\prime}\right)$.

Conversely, if $B$ is compact in $\left(X, \sigma X^{\prime}\right)$, then Theorem 2.1 shows that $\bar{B}^{\left(\mathbb{C}^{X^{\prime}}, \sigma X^{\prime}\right)} \subset X$, i.e., $\bar{B}^{\left(\mathbb{C}^{X^{\prime}}, \sigma X^{\prime}\right)}=B$. Then $X^{\prime \prime}=X$ by the Goldstine theorem $([4, \mathrm{p} .424])$.

ACKNOWLEDGEMENTS. This research was supported by Kumoh National Institute of Technology.

\section{References}

[1] A. Wilancky, Modern Methods in Topological Vector Spaces, McGraw-Hill, New York, 1978.

[2] A. Wilansky, Topology for Analysis, John wiley, 1970.

[3] J. L. Kelley, General Topology, van Nostrand, 1955. 
[4] N. Dunfont and J. Schwartz, Linear Operators I, Interscience, N. Y., 1958.

[5] W. H. Craves and W. Ruess, Compactness in spaces of vector-valued measures and a natural Mackey topology for spaces of bounded measurable functions, Contemporary Math., 2 (1980), 189-203.

[6] W. Lu and Z. Chen, General Topology, Nanjing Univ., 1995.

[7] Y. Yang and C. Swartz, A general Orliez-Pettis theorem, Stud. Sci. Math. Hung., 43(1) (2006), 47-60.

Received: January, 2013 